\title{
Clinical Study of Fungal Rhinosinusitis
}

\author{
Dr. H.S. Satish ${ }^{1}$, Dr. Jolene Alokkan ${ }^{2}$ \\ ${ }^{1}$ (Department of Otolaryngology, Bangalore Medical College \& Research Institute, Bangalore India) \\ ${ }^{2}$ (Department of Otolaryngology, Bangalore Medical College \& Research Institute,Bangalore India)
}

\begin{abstract}
Background and Objective: Over the last decade, the incidence of fungal infections has increased dramatically. This study was undertaken to study the various epidemiological and clinical risk factors associated with fungal rhino-sinusitis and to determine the best possible method for accurate diagnosis of a case

Materials and Methods: 44 cases of suspected fungal rhinosinusitis were evaluated with detailed history taking, clinical examination, haematological, histopathological and radiological investigations. Management by surgical or medical modalities were evaluated.

Results: Fungal rhinosinustis is more common in the middle aged population-particularly the $4^{\text {th }}$ and $5^{\text {th }}$ decade of life. It is also twice as common in men as in women. Patients presented with clinical features which were indistinguishable from bacterial chronic rhinosinusitis. Diabetes mellitus was found to be a major predisposing factor for the infection. CT scan had a sensitivity of $45.5 \%$ in detecting cases, while histopathology and KOH mount had a sensitivity of $90.9 \%$.

Interpretation and Conclusion: Fungal rhinosinusitis is an important clinical entity which must be considered in all patients presenting with unresolving rhinosinusitis. Early diagnosis with diagnostic nasal endoscopy and biopsy with CT scan of nose and paranasal sinuses can facilitate timely intervention in the form of surgical debridement and anti-fungal therapy in order to improve the clinical outcome.
\end{abstract}

Key word: Biopsy, CT, Diabetes mellitus, FRS, KOH mount

\section{Introduction:}

Over the last decade, the incidence of fungal infections has increased dramatically. Fungi are ubiquitous in the environment, hence we are constantly exposed to them. Resident bacterial flora probably inhibit colonization by fungi through a number of mechanisms. Thus antimicrobial therapy predisposes to both the overgrowth of normal fungal flora, e.g., Candida species, and growth of opportunists like Aspergillus ${ }^{1}$.

Fungal sinusitis should be considered in all patients with chronic sinusitis. A clear idea of the various risk factors and epidemiological profile of patients at risk of the disease and the best possible method, for early and accurate diagnosis of a case, will help ensure a successful outcome in such cases.

\section{Materials and methods:}

A prospective study was conducted on patients presenting with features suggestive of fungal rhinosinusitis to our tertiary care hospital during the study period from October, 2010 to September, 2012. Medical records of diagnosed cases of fungal rhinosinusitis were evaluated to study the epidemiological profile, risk factors, presentation and radiological features. The method of diagnosis of cases was evaluated to determine sensitivity of the technique used. All patients received surgical or medical management or combination of both. The treatment outcome of the patients was evaluated.

\section{Results:}

44 cases of fungal rhinosinusitis were diagnosed at the department of ENT between October 2010 Sept 2012. A total of 30,000 patients attended the E.N.T OPD during the same period. Of these, $6000(20 \%)$ were patients whose primary diagnosis was rhinosinusitis. The incidence of fungal rhinosinusitis overall and among patients suffering from rhinosinusitis is $7.3 \%$.

The greatest incidence of fungal rhinosinusitis was in the middle aged population with very few cases seen in the very young or the elderly. The $4^{\text {th }}$ to $6^{\text {th }}$ decade accounted for more than $80 \%$ of the cases. The disease was more common among men (29 patients) than in women (15 patients). Sex ratio was 2:1.

The most common presenting complaints of the patients at presentation were nasal obstruction, nasal discharge and chronic headache. Fungal rhinosinusitis has clinical features similar to bacterial rhinosinusitis and is hence very often undiagnosed. 


\begin{tabular}{|l|c|c|}
\hline Presenting complaint & No. of patients & $\begin{array}{l}\text { Per cent of } \\
\text { patients }\end{array}$ \\
\hline Nasal Obstruction & 39 & 88.64 \\
\hline Nasal Discharge & 36 & 81.82 \\
\hline Headache & 34 & 77.27 \\
\hline Facial swelling & 11 & 25.00 \\
\hline Visual loss & 9 & 20.45 \\
\hline
\end{tabular}

Table I: Table of most common presenting complaints of the patients

Diabetes mellitus was the most common systemic disorder associated with fungal rhinosinusitis45.45\%. High blood glucose levels are an important cause of impaired cell mediated immunity which is the primary host defence against fungal infections. Patients with uncontrolled blood sugars tended to have more aggressive infection and required prolonged hospital stay for control of infection.

\begin{tabular}{|l|l|l|}
\hline Systemic disorder & No. of patients & Per cent of patients \\
\hline Diabetes Mellitus & 20 & $45.45 \%$ \\
\hline Retro viral disease & 5 & $11.36 \%$ \\
\hline
\end{tabular}

Table II: Table showing predisposing systemic disorders in patients

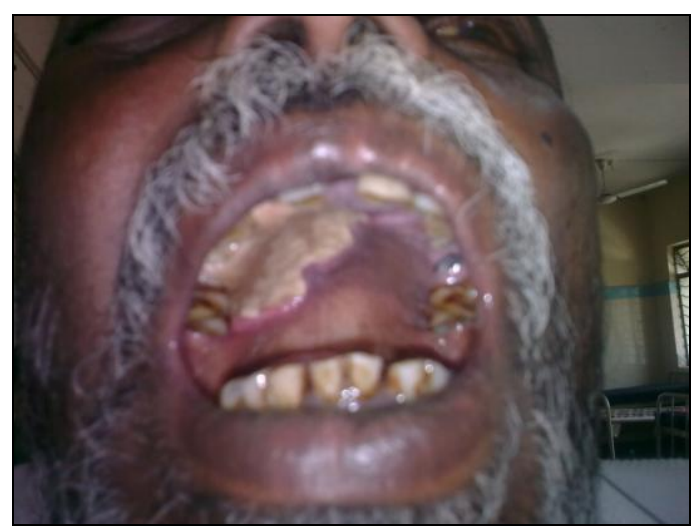

Fig. I: Picture of hard palate necrosis in a patient with right maxillary fungal rhinosinusitis

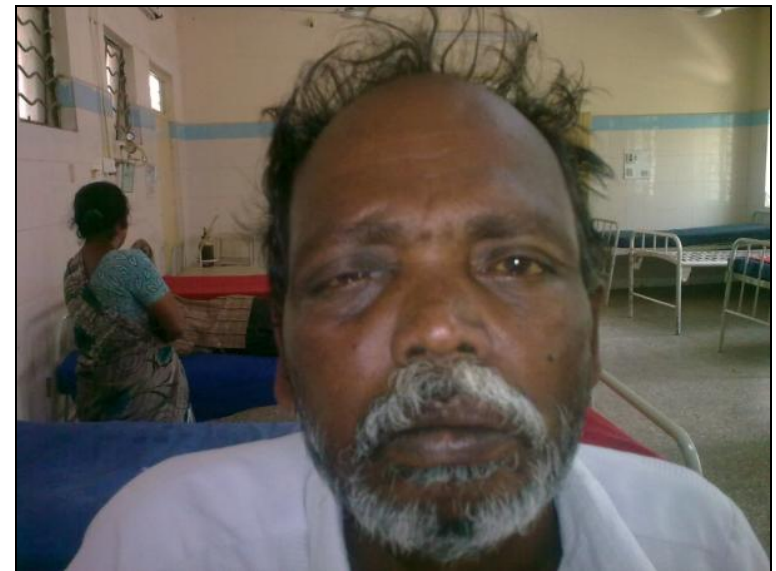

Fig II: Picture of right eye periorbital edema with displacement of the globe downwards and laterally in a patient with right maxillary and ethmoid fungal rhinosinusitis

Of the 28 patients, who underwent CT scanning as a part of radiological diagnosis, 20 had features on CT suggestive of fungal rhinosinusistis. These include -homogenous soft tissue densities with speckled calcifications, surrounding bone sclerosis, thinning of the surrounding bone and erosion. However 8 of the patients had CT scans which were not characteristic of fungal rhinosinusitis and only suggestive of non-specific rhinosinusitis.

CT scan, thus, had a sensitivity of $45.45 \%$ of detecting cases of fungal rhinosinusitis, indicating that CT scans show non-specific findings over half the time in cases of fungal rhinosinusitis. 


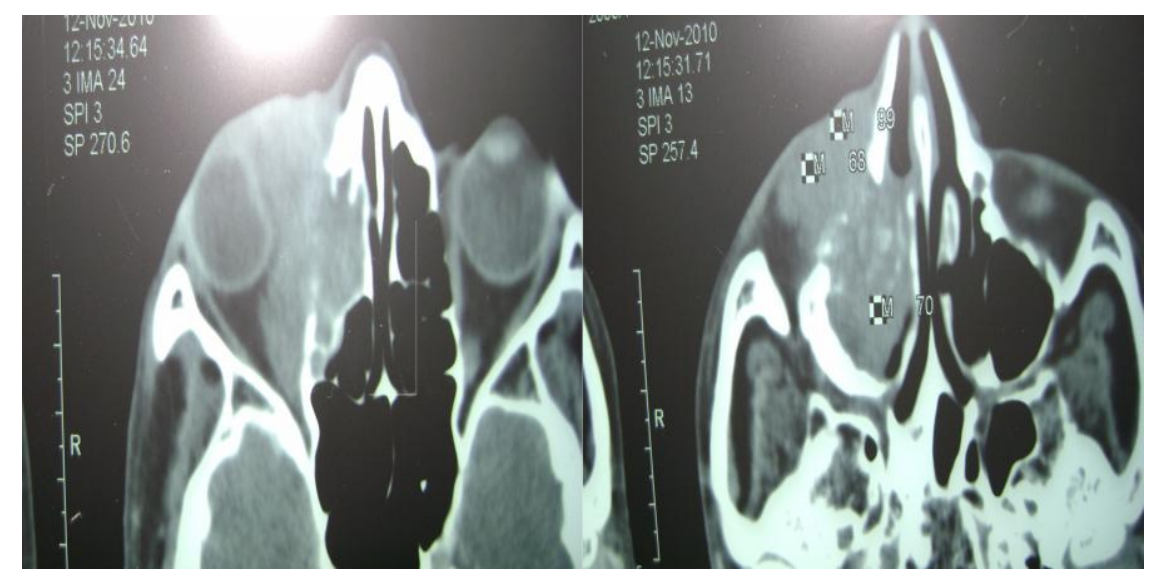

Fig. III: CT scan image showing homogenous soft tissue density in the right ethmoid extending into the orbit with intralesional speckled calcification, sclerosis of surrounding bone and erosion of lamina papyracea.

Histopathology of the fungal debris from the nasal cavity and sinuses were stained using standard eosin and hematoxylin stain. Following identification of the fungal elements, further fungal stains like GMS and PAS stains, were used to confirm the diagnosis.

Of the 40 cases that were positive for fungal elements by HPE or KOH mount, 34 showed septate fungal hyphae. The most commonest pathogenic septate hyphal fungi are aspergillus and mucor, indicating that these were the most common infecting fungi in the study group. Histopathology \& KOH mount had a combined sensitivity of $90.9 \%$ of detecting cases of fungal rhinosinusitis.

\begin{tabular}{|l|l|}
\hline & Sensitivity \\
\hline CT Scan & $45.45 \%$ \\
\hline HPE \& KOH & $90.9 \%$ \\
\hline
\end{tabular}

Table III: Table showing sensitivity of CT scan Vs HPE and KOH mount

Medical treatment with antifungals was given to 41 patients. In patients who were clinically stable and had localized disease, oral anti-fungals were prescribed. Itraconazole was the preferred drug. It was given at a dose of 200mg B.D for 6 weeks. Monitoring of renal function was done before starting and during the course of the treatment. Patients who had severe disease were admitted and given I.V amphotericin B with regular monitoring of liver and renal function.

Compliance was a major factor with antifungal therapy due to the prolonged course of treatment and cost of therapy. Surgical treatment was done with functional endoscopic sinus surgery and debridement. This was found to reduce the burden of disease and speed up recovery.

The follow-up period was minimum 3 weeks. 38 patients had complete recovery. Of the 44 cases, 6 patients died- 2 due to intracranial spread of the disease, 4 due to uncontrolled blood sugars and ketoacidosis.

\section{Discussion:}

Fungal rhinosinusitis is being increasingly recognised in persons of all age groups, resulting in great socio-economic effects, including both direct and indirect costs to the society. The impact of FRS not withstanding, the disease is often neglected and misdiagnosed especially in developing countries like India, where FRS is one among the neglected diseases ${ }^{2}$

Fungal rhinosinusitis is considered a disease of the younger age group. B. Naghibzadeh et.al. reported a mean age of $31.62 \pm 12.56$ years in their study ${ }^{3}$ and Fikret Kasapoglu et. al. reported a median age of 26 patients as 43 years (range between 9 and 74 years) ${ }^{4}$.

In this study the highest incidence of the disease was found to be in the fifth decade $33.4 \%$ with the $4^{\text {th }}$, $5^{\text {th }}$ and $6^{\text {th }}$ decade accounting for almost $80 \%$ of the cases. The youngest patient was $16 y$ rs old and the oldest patient was $65 \mathrm{yrs}$ old. The mean age was $43.81 \mathrm{yrs}$.

The diagnostic delay in fungal rhinosinusitis is often due to the clinical features of non-invasive forms of the disease resembling chronic bacterial rhinosinusitis. In this study more than $80 \%$ of the patients presented with nasal obstruction, nasal discharge and headache as their chief complaints. Ragini et. al. have also reported that the clinical presentation of most of the patients was nasal obstruction of the corresponding side, with or without headache and nasal discharge 5 .

In this study, we found 20 of the 44 patients had uncontrolled blood glucose and 5 were HIV positive with low leucocyte count. Also more than half the patients (24 out of 44) were chronic smokers. While smoking 
is known to impair ciliary activity, diabetes mellitus and retro-viral disease are known systemic disorders that cause immuno- suppression and predispose to fungal infections.

Valera FCP et.al further concluded that, the clinical outcome was associated with the underlying disease, and patients with a poorer prognosis included those with aplastic anemia and diabetes mellitus; those with an intermediate prognosis included patients with hematological diseases. Patients with AIDS/HIV or renal insufficiency presented a better prognosis ${ }^{6}$.

Fungal rhinosinusitis is a great imposter. While on one hand, certain radiologic appearances are characteristic for certain forms of the disease, yet there is a significant percentage of cases, wherein the best imaging techniques fail to clinch the diagnosis and warrant the use of nonradiological diagnostic modalities. In this study, CT scan was conclusive of fungal rhinosinusitis in only $45.45 \%$ of cases (20 of 44 cases). In the remaining cases, CT was non-specific with features of inflammation of sinus and nasal mucosa and no characteristic features suggestive of fungal rhinosinusitis.

Groppo ER et. al found that magnetic resonance imaging was more sensitive than CT for the diagnosis of FRS (sensitivity $85 \%$ ). Extrasinus invasion with MRI was the most sensitive individual parameter $(100 \%)$. Magnetic resonance imaging and CT had similar specificities, and perisinus invasion was the most specific individual parameter $(83 \% \text { for MRI compared with } 81 \% \text { for CT })^{7}$.

Evaluation of nasal debris obtained at diagnostic nasal endoscopy or surgery revealed fungal elements in 40 of the 44 cases, a sensitivity of $90.9 \%$. Most of the HPE revealed septate fungal hyphae indicative of aspergillus which is the commonest fungi in the environment in this country.

Daniel L. Hamilos found fungal elements in 54 of 54 unselected CRS cases, using silver stains. Clearly this technique has the potential to improve the diagnostic accuracy of evaluating allergic mucin for the presence of fungi, although it might prove to be overly sensitive for differentiating FRS from other cases of CRS 8 .

Thus, FRS, where early diagnosis is the key to successful management, should be evaluated by combined radiological and histopathological modalities in order to avoid both under and over diagnosis. Both false negative and false positive diagnosis should be avoided as treatment of FRS has significant implications for the patient in terms of duration, side effects and cost.

\section{Conclusion}

Patients present, most commonly, with clinical features of nasal obstruction, nasal discharge and headache, which is indistinguishable from bacterial chronic rhinosinusitis. Diabetes mellitus was found to be a major predisposing factor for the infection with uncontrolled blood glucose levels being associated with rapidly progressive disease. $\mathrm{KOH}$ mount of nasal debris is more sensitive than $\mathrm{CT}$ in diagnosing FRS. Early diagnosis and management can achieve better control over the disease.

\section{References:}

[1]. Jean Michel Klossek. Fungal Rhinosinusitis. Scott-Brown's Otorhinolaryngology, Head and Neck Surgery 7th edition. Great Britain: Edward Arnold Ltd; 2008 vol 2.p 1449-1457.

[2]. Shiv Sekhar Chatterjee, Arunaloke Chakrabarti. Epidemiology and medical mycology of fungal rhinosinusitis. Otorhinolaryngology Clinics: An international Journal; sept- dec 2009; 1 (1): 1-13

[3]. B. Naghibzadeh, E. Razmpa, Sh. Alavi, M. Emami, M. Shidfar, Gh. Naghibzadeh, A. Morteza. Prevalence of fungal infection among Iranian patients with chronic sinusitis. Acta Otorhinolaryngol Ital. 2011 February; 31(1): 35-38.

[4]. Fikret Kasapoglu, Hakan Coskun, Omer Afsin Ozmen, Halis Akalin, and Beyza Ener. Acute invasive fungal rhinosinusitis: Evaluation of 26 patients treated with endonasal or open surgical procedures. Otolaryngology-Head and Neck Surgery (2010) 143, 614-620

[5]. Ragini Tillak, Vikas Kumar, Chaitanya Nigam, Munesh kumar Gupta, Rajesh Kumar, R.K Jain. Clinicomycological Spectrum of Fungal Rhino-Sinusitis from University Hospital, North India, Journal of Clinical and Diagnostic Research. 2012 May (Suppl-2), Vol6(4): 656-659,

[6]. Fabiana C.P. Valera, Tassiana do Lago, Edwin Tamashiro, Camila Carrara Yassuda, Fla' via Silveira, Wilma T. Anselmo-Lima Valera FCP, et al. Prognosis of acute invasive fungal rhinosinusitis related to underlying disease. Int J Infect Dis (2011); 08-05

[7]. Groppo ER, El-Sayed IH, Aiken AH, Glastonbury CM Computed tomography and magnetic resonance imaging characteristics of acute invasive fungal sinusitis. Arch Otolaryngol Head Neck Surg. 2011 Oct; 137(10):1005-10.

[8]. Daniel L. Hamilos. Allergic Fungal Rhinitis and Rhinosinusitis. Proceedings of the american thoracic society VOL 72010 ; $245-252$ 